\title{
The Impact of Social Media on Negative Online Physician Reviews: an Observational Study in a Large, Academic, Multispecialty Practice
}

\author{
R. Jay Widmer, MD, PhD ${ }^{1,2}$, Margaret Shepard, $M S^{3}$, Lee A. Aase, BS ${ }^{3}$, John T. Wald, MD", \\ Sandhya Pruthi, $M D^{5}$, and Farris K. Timimi, $M D^{1,3}$
}

'Department of Cardiovascular Medicine, Mayo Clinic, Rochester, MN, USA; ${ }^{2}$ Department of Internal Medicine, Baylor Scott and White Health, Temple, TX, USA; ${ }^{3}$ Mayo Clinic Center for Social Media, Mayo Clinic, Rochester, MN, USA; ${ }^{4}$ Department of Radiology, Mayo Clinic, Rochester, MN, USA; ${ }^{5}$ Department of General Internal Medicine, Mayo Clinic, Rochester, MN, USA.

\begin{abstract}
BACKGROUND: Online reviews of physicians are becoming increasingly common, however no correlation of these reviews to formal patient satisfaction surveys. With the explosion of social media, it is unknown as to how this form of communication may have a role in potentially managing and addressing the search position of negative online reviews.
\end{abstract}

METHODS: We obtained a list of 102 physicians with negative online reviews between September 2014 and December 2014. Social media uptake and average Google search position of the physician and their respective negative online reviews were assessed from January 2015 through January 2017.

RESULTS: Fifty-four (53\%) physicians had any social media presence in January 2015. All 102 physicians were subsequently offered social media coaching by the Mayo Clinic Center for Social Media which resulted in an increase to $90 \%$ of these physicians participating in social media by January 2017. The average Google search position for the negative online reviews was significantly reduced from $5.2 \pm 2.5$ to $14.3 \pm 11.3(P<0.001)$ from 2015 to 2017 . There was a moderate increase in Doximity uptake during that time increasing from $11 \%$ of the physicians having a claimed profile to $80 \%$. There were nonsignificant reductions in the average Google search position $-9.7 \pm 11.3$ in the physicians who had an existing a social media presence versus those who were not on social media $-4.2 \pm 2.2(P=0.11)$.

CONCLUSIONS: Physician social media presence can reduce the bearing of negative online comments by decreasing the search position of these comments.

KEY WORDS: social media; physician reviews; online; internet.

J Gen Intern Med 34(1):98-101

DOI: $10.1007 / \mathrm{s} 11606-018-4720-3$

(C) Society of General Internal Medicine 2018

Received July 25, 2018

Revised August 30, 2018

Accepted October 19, 2018

Published online October 29, 2018

\section{INTRODUCTION}

Online physician reviews are increasingly pervasive in healthcare, ${ }^{1}$ despite their lack of correlation to formal institutional surveys ${ }^{2}$ or reflection of overall clinical outcomes. ${ }^{3}$ These comments can negatively affect a physician's online reputation. Indeed, our recent work has demonstrated that negative online reviews do not correlate with formal patient satisfaction reviews. ${ }^{4}$ It is unknown what methods are most efficacious in countering these negative online reviews on various websites on the intranet.

Over the last decade, we have seen a substantial increase in the use of social media platforms in general and even more so in the healthcare setting. While most of the published literature on the use of social media by physicians is focused on anecdotes and risk aversion, our group demonstrated the positive impact of initiating a Twitter account by cardiology fellows and the potential to reach wide swaths of heart failure patients through Facebook and YouTube. ${ }^{5}$ As social media adoption continues to expand in healthcare, it is unclear the potential role this vehicle could play in ameliorating the effects of negative online reviews of physicians.

In recognition of the rise of online reviews, physician brand management has become an emerging concern for physicians. Despite this recognition, many physicians remain reticent to fully understand the important issues, let alone take preemptive measures to actively manage their brand - particularly in the strategic exploration of online tools to include social media. The emergence of service-centered businesses has allowed for a co-creation of a bidirectional exchange between consumers and suppliers. ${ }^{6}$ However, there are no concrete data to point to the value of such co-created online relationships, and thus the space for online brand management for physicians has largely been ceded to marketing companies that attempt to impact these relationships using automated social media accounts and search engine optimization techniques. While there is a notion that these metrics can be manipulated and impacted by emphasizing search prioritization on external features, i.e., links from other sites, search algorithms are now increasingly limiting webmasters to "game" search engine results via changes in their own websites. Despite the 
unknown overall impact of Internet search position-particularly regarding both positive and negative online reviews - on brand management, there is a growing interest in the ability to use social media tools to potentially optimize website and individual online presence. ${ }^{7,8}$

The aim of this study was to evaluate how a dedicated social media intervention for physicians who have negative online reviews could change the search profile of their online presence over time. We hypothesized that those with an increased social media presence would see a decrement in the Google position of their initial negative review.

\section{METHODS}

We utilized a cohort of physicians with negative online reviews based on a previous publication. ${ }^{4}$ Briefly, institutionwide Google Alerts from September 1, 2014, through December 31,2014, were used to track negative online reviews of physicians at Mayo Clinic's Rochester, MN campus in Rochester, Minnesota. Negative online reviews were classified as any ratings below $50 \%$ on a numerical scale, ratings below "C" on a letter scale, or negative associated comments. These negative reviews were then assembled and passed on to the patient experience leadership staff, the Mayo Clinic Center for Social Media (MCCSM), and the departmental/division chairs. Those with a negative online review were offered education from the MCCSM regarding individual social media opportunities and platforms with subsequent instruction on how to enhance their online presence. This included face-toface instruction on the basic functions and use of Twitter, Doximity, LinkedIn, and YouTube, and online training via the Social for Health Care Certifications CME course. ${ }^{9}$ Doximity, Inc. is an online social networking site specifically for physicians and midlevel providers launched in 2011 now with over one million members (approximately $70 \%$ of US physicians, $45 \%$ of physician assistants and nurse practitioners, and $90 \%$ of US fourth-year medical students). Doximity provides curated medical news, HIPAA-compliant case sharing capabilities, and secure messaging for physicians across multiple medical specialties and centers. ${ }^{10}$

We identified 141 negative online reviews among 113 individuals. Eight "non-physicians" (dentists, nurse practitioners, physician assistants, and psychologists) were excluded, as were three physicians who did not have their negative online reviews positioned within the first five pages of a Google search. This resulted in an overall cohort of 102 physicians with at least one negative online review, and 16 of these individuals had greater than one online reviews.

The social media presence of each of these physicians was evaluated and documented in January 2015. We recorded the presence of a Twitter account, claimed Doximity profile (must include photograph), LinkedIn profile, and presence of a YouTube video. We also recorded the Google search position of the negative online review in January 2015 and January
2017. Social media presence on Twitter, Doximity, LinkedIn, and YouTube was again assessed in January 2017. The primary outcome of the study was to assess the change in Google search position between January 1, 2015, and January 1, 2017. Secondary outcomes included participation of various social media platforms (Twitter, Doximity, LinkedIn, and YouTube) and individual change in Google search position within the various social media platforms.

Data analysis was completed using downloaded raw metrics from Excel to JMP, a statistical software package (version 9.0, SAS Institute Inc.). For continuous data, results were summarized as means and 95\% CIs. Means \pm SDs were calculated and presented. Two-sample $t$ tests were used to compare continuous scores between groups; chi-square tests were used to compare categorical results between groups. A conventional two-sided $\alpha$ level of 0.05 was used for all data to determine statistical significance.

\section{RESULTS}

Of the 102 eligible physicians, only $54(53 \%)$ had a social media presence in 2015 compared to 91 (90\%) in 2017. This cohort included physicians from 20 medical departments (83 physicians) and 8 surgical departments (19 physicians). Sixtyseven $(66 \%)$ were male. Initial social media use in 2015 and then in 2017 is reported in Table 1. There was a significant reduction in average Google search position of the negative online comment in the two years $(-9.1 \pm 10.8, P<0.001 *)$ and percentage of negative reviews moved off the first Google search page ( $4 \%$ vs $55 \%, P<0.001 *$ ).

Those who had prior participation with online social media efforts had non-significant reduction in the average Google search position of their highest ranking negative online review $-9.7 \pm 11.3$ compared to those participated in the social media classes offered by MCCSM $-4.2 \pm 2.2(P=0.11)$. Similarly, in those with Google search position > 10 (off the first Google search page), there was a non-significantly greater proportion of social media uptake (69 physicians (62\%) vs 43 physicians (38\%); OR $2.3(0.8,6.5) P=0.11)$.

Except for Twitter, the other three social media platforms all demonstrated the capacity to be positioned higher in Google

Table 1 Changes in Social Media Platform Use 2015 to 2017

\begin{tabular}{lll}
\hline \hline & $\mathbf{2 0 1 5}$ & $\mathbf{2 0 1 7}$ \\
\hline Any social media? & $54(53 \%)$ & $91(90 \%)$ \\
Twitter & $9(9 \%)$ & $12(12 \%)$ \\
Doximity & $\begin{array}{l}\text { Yes, 64 (63\%) } \\
\text { "Claimed," } 11\end{array}$ & $\begin{array}{l}\text { Yes, } 20(20 \%) \\
\text { "Claimed," } 82(80 \%)\end{array}$ \\
$\begin{array}{l}\text { LinkedIn } \\
\text { YouTube }\end{array}$ & $23(23 \%)$ & $40(40 \%)$ \\
$\begin{array}{l}\text { Average search } \\
\text { position }\end{array}$ & $33(32 \%)$ & $26(25 \%)$ \\
$\begin{array}{l}\text { Negative review off } \\
\text { first Google page? }\end{array}$ & $4.2 \pm 2.5$ & $14.3 \pm 11.3 *$ \\
\hline$*<0.05$ & & $55(54 \%) *$ \\
& &
\end{tabular}


Table 2 Change in Search Position from 2015 to 2017, and the Adoption of Various Social Media Platforms

\begin{tabular}{llll}
\hline \hline $\begin{array}{l}\text { 2017 position } \\
\text { change }\end{array}$ & Non-user & Established user & New user \\
\hline Twitter & $-8.5 \pm 1.1$ & $-17.1 \pm 3.5$ & $-2.3 \pm 6.1$ \\
Doximity & $-8.5 \pm 1.8$ & $-11.0 \pm 4.1$ & $-9.3 \pm 1.4$ \\
LinkedIn & $-9.9 \pm 1.4$ & $-5.3 \pm 2.5$ & $-10.1 \pm 2.3$ \\
YouTube & $-7.3 \pm 1.2$ & $-13.7 \pm 2.5$ & $-15.7 \pm 3.5$ \\
\hline
\end{tabular}

search ahead of the individual physician's negative online review. The mean jump in Google search position for Doximity was $5.4 \pm 3.5$, for LinkedIn was $4.5 \pm 3.9$, and was $5.2 \pm 6.4$ for YouTube. Table 2 highlights changes in Google search position for individual social media platforms. Despite improvements in Google search positions among the groups, there were similar reductions in Google search positions among those who did not use social media, those who had adopted social media prior to 2015 , and those who participated in various social media platforms between 2015 and 2017.

\section{CONCLUSIONS/DISCUSSION}

Here we leveraged an opportunity to address ways to manage and enhance physician online social media presence. Our previous work demonstrated the lack in correlation between negative online physician reviews and formal patient satisfaction surveys. With social media education and instruction, a substantial number of physicians were able to more effectively manage and address their negative online reviews and show a drop in the ranking of these reviews. These results highlight a significant uptake of over $90 \%$ of physicians toward various social media platforms - predominantly simply claiming a Doximity profile - and a concomitant reduction in the Google search position of the negative online reviews in the two years. Although there could be a time-dependent component to the reduction in search position in these negative online reviews, these data demonstrate how enhancing one's social media presence can improve their online reputation.

Over a two-year period, there was a $66 \%$ increase in social media presence leading to a nearly twofold reduction in Google search position among those physicians who had a negative online review. The most notable social media participation was in those physicians who simply "claimed" their Doximity profile (11\% increased to $80 \%$ ). There was only modest uptake in the social media platforms such as Twitter and LinkedIn, and a variable presence on YouTube.

We chose to assess the influence of the negative online review using Google search position. While this metric could have flaws based on geofencing, time of day, and other search algorithm confounders, there are currently no established metrics regarding most physicians' online presence. The fact that most negative reviews dropped off the first Google search page is notable. Most Internet users do not search beyond the first Google page, and thus these poorly monitored and non-validated reviews can be essentially buried in cyberspace and less likely to directly negatively affect the physician's online reputation. Although we did not characterize the Google search results for non-social media sites, it is possible that other institutional web pages could mitigate the presence of the negative online review. It cannot be discounted that simply time alone could be the most important factor in lowering the ranking of the negative reviews on the web page. However, study highlights a proactive way to manage a physician's online reputation. Individual social media engagement remains one of the only active methods that could be used to reduce the Google search position of a negative online review.

A limitation of the study is the non-randomized, retrospective cohort without a comparator group. Further well-designed prospective studies could provide more information regarding the potential positive impact of social media in mitigating the adverse effects of these negative online reviews. This follow-up study of our previous work was underpowered to detect significant differences among such heterogeneous data using multiple comparisons. For these reasons, one must be careful to not overemphasize the effect of social media on negative online reviews. However, a comprehensive and structured social media educational program for physicians lays the groundwork for further study and exploration into efforts to improve one's online presence. Finally, it should be noted that there were no adverse events stemming from participants' social media interventions, and social media engagement did not exacerbate the negative online reviews.

This study provides initial evidence for an association between a social media intervention on physicians with reported negative online reviews. These are some of the first data to demonstrate a significant increase in social media uptake among physicians with negative online reviews and concomitant reduction in Google search position of bespoke negative online reviews. Although there was not one specific social media platform that had the greatest influence, there was no obvious negative affect stemming from this intervention. Further prospective work on the impact of social media on the online reputation of physicians should be explored.

Acknowledgements: The authors would like to thank Michelle Rinn and Janine Kamath for coordinating the Google searches and Google alerts. We would also like to thank Unnikrishnan Gopinathan for his assistance with coordinating and organizing the raw patient satisfaction survey data.

Corresponding Author: R. Jay Widmer, $M D, P h D$; Department of Internal Medicine Baylor Scott and White Health, Temple, TX, USA (e-mail: robert.widmer@BSWHealth.org).

\section{Compliance with Ethical Standards:}

Conflict of Interest: The authors declare that they do not have a conflict of interest.

\section{REFERENCES}

1. Emmert M, Sander, U, Pisch, F. Eight Questions About PhysicianRating Websites: A Systematic Review. J Med Internet Res. 2013;15(2):e24.

2. Ryan T, Specht, J, Smith, S, DelGaudia, JM. Does the Press Ganey Survey Correlate to Online Health Grades for a Major Academic 
Otolaryngology Department? . Otolaryngol Head Neck Surg. 2016;155(3):411-5.

3. Okike K, Peter-Bibb, TK, Xie, KC, Okike, ON. Association Between Physician Online Rating and Quality of Care. J Med Internet Res. 2016;18(12):e324.

4. Widmer R, Maurer, MJ, Nayar, VR, Aase, LA, Wald, JT, Kotsenas, AL Timimi, FK, Harper, CM, Pruthi, S. Online Physician Reviews Do Not Reflect Patient Satisfaction Survey Responses. Mayo Clin Proc. 2018;93(4):453-7.

5. Widmer R, Arce, MK, Aase, LA, Timimi, FK. Social media platforms and heart failure. J Card Fail. 2017.
6. Hsieh P. Encounters in an online brand community: development and validation of a metric for value co-creation by customers. Cyberpsychol Behav Soc Netw. 2015;18(5):286-95.

7. Zhang SaC, $\mathbf{N}$. Search Engine Optimization: Comparison of Link Building and Social Sharing. J Comput Inf Syst. 2016;57(2):148-59.

8. Jones S. A Re-Examination of Fortune 500 Homepage Design Practices. IEEE Trans. 2015;58:20-44.

9. Available from: https://healthcare.hootsuite.com. Accessed Sept 2018.

10. Buro J. App Review Series: Doximity. J Digit Imaging. 2018. 